\title{
NOTAS SOBRE EL DERECHO CANÓNICO Y LAS FUENTES ECLESIÁSTICAS EN EL PENSAMIENTO DE FRANCESC EIXIMENIS
}

\author{
MANuel J. PeláEZ \\ Universidad de Málaga
}

La obra de Francesc Eiximenis, quien contaba con una preparación filosófica y teológica que tuvo oportunidad de ampliar en la Universidad de Toulouse, presenta, sin embargo multitud de referencias al Derecho Canónico como derecho de la Iglesia', y una familiarización con las obras

\begin{abstract}
'Dedicamos estas breves páginas a Regina Sáinz de la Maza Lasoli. No hemos querido estar ausentes en su homenaje, siendo persona con la que tuvimos oportunidad de convivir en aquel fecundo Departamento de Estudios Medievales de la Institució Milà i Fontanals del Consejo Superior de Investigaciones Científicas de Barcelona bajo la tutela del prof. Emilio Sáez Sánchez, igualmente desaparecido de triste forma. Mucha gente no sabe en España la deuda de gratitud que la investigación histórica tiene con ese hombre, en la organización. en la promoción, en la animación, en la financiación y en muchas otras cosas. Pero volviendo a Regina. me permito recoger alguna idea por la vía de anecdotario. Era una persona seria. No le oi nunca hacer bromas, ni comentarios jocosos, ni con las personas a cuenta de la ciencia o las investigaciones que se llevaban a cabo en el CSIC. No era mordaz, ni ácida con las investigaciones ajenas. Presumo que sería católica, y no sólo por su contacto directo con las instituciones eclesiásticas como tema de investigación permanente, sino por algo que sucedió con ocasión del fallecimiento de Albino Luciani, hasta entonces felizmente reinante como pontífice Juan Pablo I. Llegó al Consejo aquella mañana dolorida. El comentario que nos hizo -era ya el mes de setiembre de 1978 - fue claro: «me he quedado helada. Se me han puesto los pelos de punta. Pobre hombre ¡Qué pena!». Vi los ejercicios que realizó a la plaza de Colaboradora Científica de Historia Medieval, y cómo logró imponerse ante otra candidata nada fácil, pertrechada de una magnífica tesis doctoral y de estancias investigadoras en el extranjero a uno y otro lado del Atlántico. La solidez de sus monografïas, su reconocimiento científico internacional y su bonhomía (mejor sería decir su "bonmuliería", que no lo admite la Academia) era algo patente en su comportamiento. Mujer atractiva, elegante y con caché. quizás algo distante, erguida, no inclinaba el cuerpo ni siquiera para mirar los ficheros de la sala de consulta general donde trabajaba la eficacísima Rosa Mayordomo y enfrente unos cuantos investigábamos sobre otras cosas haciendo ver que escribiamos voces del famoso Potthast (quizás con la sola excepción de Jesús Fernández Viladrich, que ese sí se lo tomó más en serio, y de un tal Alberto Martínez, no así Josep Maria Sans i Travé, ni Jordi Rius Cornadó, ni yo). Observé en ella un
\end{abstract}


de Graciano, del Cardenal Hostiense y de no pocos decretistas y decretalistas, como Simón de Tournais o Guillaume de Auxerre y no digamos con el caso particular de Bonifacio VIII por medio de sus conocidas Bulas en su polémica con el rey de Francia.

El Decretum o Concordia discordantium canonum es instrumento de trabajo de Eiximenis y de rigurosa innovación en numerosos pasajes de sus obras, no sólo en las de contenido jurídico-político como el Dotzè del Crestià, el Regiment de la cosa pública ${ }^{2}$ o la Doctrina compendiosa (si es que esta última obra es original de Eiximenis), sino también en otros lugares y pasajes del Primer, el Segon o el Terç del Crestià, aparte del Libre de les dones. Frecuentemente algunos de los textos de los Padres de la Iglesia son tomados indirectamente del Decretum, no de sus fuentes originales. De esta obra la Causa XXIII ha influido en la redacción del Dotzè del Crestiă ${ }^{3}$ y del Terç del Crestià.

cierto rictus de tristeza en los últimos años -en apreciación pasajera- que no sabría a qué atribuirlo, quizás al calvario de la gravísima y prolongada enfermedad de su cuñado Josep Trenchs i Odena, un hombre meritorio en tantos sentidos y con una dedicación a la investigación científica como no he visto cosa igual. Oyéndole hablar en una oportunidad en la que formaba parte de un Tribunal de una tesis doctoral fue cuando comprendí que la Paleografía y la Diplomática no eran materias instrumentales o meramente auxiliares de la Historia. Assombrado quedé de tanto tecnicismo. A ella, la Providencia, que sabe como actúa y cuyos designios son inescrutables, la golpeó de forma rápida y sin sufrimientos. Sin duda, porque estaba preparada, porque Dios siempre sabe más.

'Dos trabajos de Donna M. Rogers, Legal Terms in Eiximenis Regiment de la cosa puiblica, en "Revista de llengua i dret", 15 (1991), pp. 35-41, y La llengua oral al Regiment de la cosa pública de Francesc Eiximenis, en "Actes del Onzè Col.loqui Internacional de Llengua i Cultura Catalanes”, Palma de Mallorca, 8-12 septiembre 1997, ed. Abadía de Montserrat-Barcelona, 1998, pp. 431-438, no aportan nada de interés. Al menos así me lo parece. Quizás a Rogers no. Su comunicación al Congreso de Palma es elemental, sin notas y con escasa bibliografía.

${ }^{3}$ Dotzè del Crestiò, Va 512, fol. 63va, se inspira en Decretum, c. 34. C. XXIV, q. 1; Dotzè, Vil 353, fol. 218rb en Decretum, c. 3, C. XXIII, q. 1 y c. 48, C. XXIII, q. 5: Dotzè, Va 654, fol. 219ra en Decretum, c. 3. C. XXIII. q. 1: Dotze, Va 654, fol. 219rb en Decretum, c. 1. C. XXIII, q. 1; Dotzè, Va 655, fol. 219rb y Va 658, fol. $222 \mathrm{vb}$ en Decretum, c. 4, C. XXIII. q. 1; Dotzè, Va 657, fol. 221 va en Decretum, c. 1. C. XXIII, q. 2; Dotzè, Va 657, fol. 222 ra en Decretum de Ivo DE ChARTRES, XII, 22 y X, 97 y Decretum, c. 2, C. XXIII, q. 2 y c. 5, C. XXIII, q. 3; Dotzè, Va 658, fol. $222 \mathrm{vb}$ en Decretum, c. 3, C. XXIII, q. 1; Dotzè, Va 658, fol. 223va en Decretum, c. 1. C. XXIII. q. 8; Dotzè. Va 659, fol. 223va en Decretum. c. 25, C. XXIII, q. 5; Dotzè, Va 660, fol. 224rb en Decretum, c. 4, C. XXIII, q. 1; Dotzè, V a 660, fol. 224va en Decretum, c. 99, C. XI, q. 3; Dotzè, Va 662, fol. 225ra en Decretum, c. 2, C. XXIII, q. 2; Dotzè, Va 663, fol. 225rb en Decretum c. 17, C. VI, q. 1; Dotzè, Va 663, fol. 225rb en Decretum, c. 3-7, C. XXIII, q. 1: Dotzè, Va 663, fol. 226ra en Decretum, c. 7. C. XXIII, q. 3 y c. 25 , C. XXIII, q. 5; Dotzè, Va 663 , fol. 226 ra en Decretum de Ivo DE CharTRES, X, 120; Dotzè, Va 666, fol. 228rb en Decretum, c. 7, C. XXIII, q. 3: Dotzè, Va 667, fol. 229ra en Decretum, c. 26. C. XXIII, q. 8: Dotzè, Va 667, fol. 229ra en Decretum de Ivo DE CharTRES, XIII. 114 y en Decretim, c. 11. C. XXIII, q. 8: Dotzè, Vil 668. fol. 230rb en Decretum, c. varios. C. XXIII. ч. 8 y c. 2. C. XXIII, q. 3. 
La obra de Tito Livio $A b$ Urbe condita había sido traducida al francés por Pierre Bersvire entre los años 1354 y 1359. El monarca catalán Juan I solicitó que le fuera remitida una copia de la traducción francesa a través del duque Jean de Berry. De esta versión y del texto latino se hizo una traducción catalana, probablemente por parte de Guillem de Copons que también había traducido el Tesoro de Brunetto Latini de la que se conserva un buen manuscrito en el British Museum, Ms. Harley, $n^{\circ} 4893$ y sobre el que el profesor de la Universidad de Saskatchewan en Saskatoon, Curt J. Wittlin, uno de los máximos especialistas en Francesc Eiximenis, elaboró una edición en dos volúmenes. Francesc Eiximenis conoció muy probablemente el original francés de la obra de este abogado florentino que había sido maestro de Dante; sin embargo, no pudo conocer la versión catalana de Copons, habida cuenta de que se terminó con casi total probabilidad siete años después de la muerte de Eiximenis. Diversos comentarios medievales a la Ética a Nicómaco de Aristóteles ${ }^{4}$ también fueron manejados por Eiximenis. Presumo que el conocimiento que tiene del Codex, de las Tres Partes, del Digestum Vetus, del Novum, del Infortiatum y de las Institutiones es indirecto, habida cuenta de la precariedad y obscuridad con que se mencionan estos textos en las obras de Eiximenis, sin concreción de ningún tipo, lo que no ocurre con los textos canónicos. A pesar de lo que escribí en otra ocasión ${ }^{5}$, no tengo nada claro ahora que Eiximenis conociera los escritos de los juristas de la Escuela de Orléans, Jacques de Révigny y Pierre de Belleperche, ni tampoco que tuviera en sus manos ninguna obra de Goffredo da Trani.

El Tractatus de pace de Marie de Dacupierre pudo ser consultado por Eiximenis como otras obras de Odon de Rigaud y Francisco Mairon.

\footnotetext{
${ }^{4} \mathrm{Ha}$ sido mencionada la influencia de Aristóteles en el pensamiento de Eiximenis por diversos autores. Ya se hizo eco de ella Josep PUIG I CADAFALCH, Idees teòriques sobre urbanisme en el segle XIV: Un fragment d'Eiximenis". en "Homenatge a Antoni Rubió i Lluch", en los "Estudis Universitaris Catalans", XXI (1936), pp. 1-9, particularmente, pp. 8 y 9. En Lo libre de les dones, ed. Frank NacCARATO, Curt WitTLin y Antoni Comas, Barcelona, 1981, hay menos referencias a Aristóteles de las que cabía pensarse (cap. 14, p. 28; cap. 17, p. 34; cap. 68, p. 108 ; cap. 72 , p. 114 ; cap. 80 , p. 124; cap. 152 , p. 230).

${ }^{5}$ Las fuentes literarias, teológicas, jurídicas, políticas y canónicas de las obras de Francesc Eiximenis, en Ralph NELSON ET ALII, "Papers in Public Law, Public Legal History, Natural Law and Political Thought. Estudios en homenaje al profesor Jesús Lalinde Abadía”, Barcelona, 1992. p. 233.
} 
John of Wales ${ }^{6}$ ha dado lugar a algunas observaciones hechas en relación a la incidencia de su obra en el pensamiento de Eiximenis, sobre todo por lo que se refiere a la versión catalana del Breu parlament de les virtuts del antichs philòsophs. La traducción de esta obra se llevó a cabo durante el reinado de Pedro el Ceremonioso y fue manejada por Eiximenis. Cuando el gerundense hace mención de algunas anécdotas ocurridas en Oxford, probablemente sean referencias indirectas tomadas de la obra de este doctor en teología quien enseñó en el colegio del que disponían los frailes minoristas en la culta ciudad inglesa. John of Salisbury a través de su Polycraticus fue manejado por Eiximenis?

El derecho canónico contenido igualmente en las Partidas lo conoce Eiximenis por medio de la traducción catalana de Mateu Adrià. Un texto jurídico de derecho feudal como el Libellus de batailla facienda fue reproducido por Eiximenis en el Dotzè del Crestià entre los capítulos 323, 324, 325, 326, 327, 328, 329 y 330.

Ramon de Penyafort, compilador de las Decretales de Gregorio IX, no es citado por Eiximenis ${ }^{8}$; no obstante, sí lo fue el canonista Juan Andrés, e igualmente Martín de Tropau, dominico, que fue nombrado por Nicolás III obispo de Gniezno en 1279. Es el autor de una obra importante de crónica de pontífices y emperadores y de la denominada Margarita Martiniana o Tabula Decreti (se refiere al Decretum de Graciano). Lo conoce Eiximenis como Martín "el polaco" (Martí, lo polonés).

Su familiarización con las instituciones eclesiásticas le viene por su condición de minorista franciscano, por su presencia en Valencia y en Barcelona y por sus relaciones con Benedicto XIII que le elevó al episcopado, siendo consagrado el 15 de noviembre de 1408 por parte del cardenal Jean d'Armagnac'. Igualmente, su estancia en Montpellier le permitiría un

\footnotetext{
${ }^{6}$ Ver Curt J. Wittuin, La Summa de Colacions de Juan de Gales en Cataluña, en "Estudios Franciscanos", LXXII (1971), pp. 190-196; Conrado GUARDIOLA, Juan de Gales, Cataluña y Eiximenis, en “Antonianum”, LXIV (1989), pp. 330-365.

${ }^{7}$ Ver Albert G. Hauf I Valls, Eiximenis, Joan de Salisbury $i$ Joan de Gal-les, en "Miscel-lània Sanchis Guarner", Abadia de Montserrat-Barcelona, 1992, pp. 239-262.

${ }^{8} \mathrm{~A}$ pesar de lo que sugieren a título de hipótesis David J. VIERA y Jordi PIQUÉ, La dona en Francesc Eiximenis, Barcelona, 1987, p. 16.

${ }^{9} \mathrm{Hay}$ la vieja referencia del libro de Pierre PUIGGarí, Catalogue biographique des évêques d'Elne, Perpignan, 1842, p. 69. Se puede completar con Jill R. WEBSTER, Nuevas aportaciones a los estudios eiximenianos. Francesc Eiximenis, OFM: su familia y su vida, en "Archivo IberoAmericano", XXXIX (1979), pp. 429-435. A Eiximenis le sustituiría en la sede de Elna Alfonso de Tous, que era un protegido de la monarquía catalano-aragonesa. El Papa Luna el 15 de mayo
} 
conocimiento no sólo de las instituciones eclesiásticas sino también de la Facultad de Derecho del prestigioso Studium generale, donde tiene oportunidad de criticar ampliamente a los estudiantes de leyes civiles y de cánones, simulando la presencia de un campesino admirado porque tantos estudiantes se dedicasen al estudio del Derecho y luego pudieran llegar a ser abogados. Para él si uno solo había originado grandes estragos entre la población civil de su lugar de residencia, se echaba las manos a la cabeza de lo que podrían llegar a ser tantísimos «defensores de la ley». No está claro que Eiximenis tomara parte en las sesiones del Concilio celebrado en Perpignan a partir del 15 de junio de 1408, habida cuenta de que su fallecimiento se produce el 23 de enero de 1409 y el concilio se mantuvo hasta el 26 de marzo. Lo que sí es seguro son las posiciones favorables al papado de Avignon que adoptó dicho Concilio. Si embargo, su presencia en dicha asamblea eclesiástica le fue requerida por el propio Pedro de Luna. Sabemos su posición favorable al papado aviñonés lo que se deduce no sólo de un comentario que hace en el Primer del Crestià (V $\left.{ }^{\mathrm{a}}, 245\right)$. Ahora bien, en la obra De triplici statu mundi hay una referencia indirecta a Avignon que se nos antoja como no suficientemente clara («in istis enim temporibus sol oscurabitur et verus Papa occultabitur... et Luna non dabit lumen suum») y algunos la han interpretado como si se tratara de un posicionamiento en defensa de Urbano $\mathrm{VI}^{10}$.

de 1409 comunicó a Martín el Humano dicho nombramiento. Ver Joan de Déu BANCHS DE NAYA, Aportación al estudio de Benedicto XIII (1394-1423), tesis de licenciatura parcialmente inédita, Facultad de Filosofía y Letras, Universidad de Barcelona, 1971, pp. 134-136, n 16.

${ }^{10} \mathrm{El}$ De triplici statu mundi se conserva en un códice de la Biblioteca de El Escorial, ms. H-III-24, fols. 198r-206v. El descubridor del ms. fue A. LóPEZ, Codicografía catalana, en "Estudios Franciscanos", III (1909), pp. 21-23. Sobre su autenticidad las opiniones están divididas. El P.A. IVARS (El terratremol de l'any 1396 en València i son regne, en "Diario de Valencia”, 21 de abril de 1915) creía que esta obra era original de Eiximenis. Otros negaron su autoría como J. M. Pou, con ciertas reservas. Fue, sin embargo, el P. Martí de BarCELONA quien más se opuso [Fra Francesc Eiximenis, O.M (1340?-1409?). La seva vida. Els seus escrits. La seva personalitat literària, en "Estudios Franciscanos", XL (1928), pp. 486-488]. Por de pronto restaba como poco firme la atribución a Eiximenis del manuscrito por la defensa que hacía de Urbano VI. Sin embargo, el propio códice reconocía como autor a Eiximenis y allí se describe el terremoto valenciano del 18 de diciembre de 1396, cuando entonces vivía el franciscano en la ciudad del Turia. Hay signos apocalípticos similares que aparecen en otras obras de Eiximenis, como Qüestions dels novissims y Vida de Jesuchrist. Albert G. HaUf I VALLS ha editado y anotado el texto con posterioridad a todos los que antaño habíamos estudiado el manuscrito, y lo hizo anunciando para una posterior entrega los criterios convincentes y definitivos sobre la paternidad del De triplici statu mundi del minorista $[E l$ "De Triplici statu mundi" de Fr. Francesc Eiximenis, O.F.M., en "Estudis Universitaris Catalans. Estudis de llengua i literatura catalanes oferts a R. Aramon i Serra en el seu setantè aniversari", XXIII (1979), pp. 265-283]. 
El pontificado de Bonifacio VIII (1294-1303) es objeto de algunas reflexiones en relación al mismo por parte de Eiximenis. Son las tres grandes bulas de este pontífice las referentes al poder directo del Papa las que sugieren algunos comentarios de Eiximenis. La rotundidad con que el pontífice considera que los laicos no tenían autoridad alguna sobre los clérigos y, en general, sobre las personas y bienes de la Iglesia es otro de los temas objeto de comentario en los escritos del gerundense ${ }^{11}$. El asunto de la obediencia a la autoridad eclesiástica o el de los subsidios y ayudas a la Iglesia, junto a la idea de lo que se entiende por bienes eclesiásticos en el sentido estricto del término, es otra de las materias objeto de consideración por parte del minorista en sus observaciones sobre el pensamiento y la actuación del pontífice romano. Es a través de la bula Etsi de statu (1297) como el Papa autorizó al rey francés a asumir algunas posiciones concretas en los supuestos de minoría de edad. Con esta bula Bonifacio calmó al monarca galo, pero él recibió la amenaza de nuevas penas de excomunión si se apropiaba indebidamente del dinero que pertenecía a la Iglesia como fruto de distintas limosnas dirigidas no sólo a la Iglesia en sí misma considerada, sino de cara a los santos lugares.

No es conveniente que ningún cristiano y mucho menos los príncipes católicos cuestionen la autoridad papal, pues si un pontífice hace una decretal y la autoridad civil no la obedece, ello ocasionará un perjuicio notable a la comunidad política y al bien público ${ }^{12}$. Es conveniente crear un animus oboediendi al pontífice, porque todos los príncipes cristianos son tributarios y vasallos del Papa conforme a la concepción de Bonifacio VIII y no sólo por aquello que establecían las viejas leyes del Antiguo Testamento de que quien no desee obedecer al sacerdote soberano merece la muerte. En otra ocasión, Bonifacio con su Bula Periculosos trató de corregir la actitud indisciplinada y disoluta de algunos clérigos, en un momento en el que la sociedad clerical y en particular el mundo franciscano atravesaba por ciertas conmociones. Es a través de la obra Ars praedicandi populo que Eiximenis se dirige al mundo clerical tratando de establecer los criterios propios de la

\footnotetext{
"Aquí el tema de referencia es la Bula Clericis laicos, sobre la que ha escrito interesantísimas páginas L. SanTIFALLER, Zur original Überlieferung der Bulle Papst Bonifaz. VIII, Clericis laicos von 1296 Februar 25, en "Studia Gratiana", XI (1967), pp. 69-90.

${ }^{12} \mathrm{Ver}$, con un carácter general, el artículo de J. LECLERC, Questions des XIII et XIV siècles sur la jurisdiction de l'Eglise et le pouvoir séculier, en "Studia Gratiana", XII (1967), pp. 309324.
} 
predicación. Esta era una obra desconocida entre los estudiosos de Eiximenis hasta que Martí de Barcelona ${ }^{13}$ la descubrió en un manuscrito en Polonia. Después no se ha puesto en duda por la crítica la autenticidad de dicha obra. En la misma se perfilan los rasgos de las obras latinas del franciscano, aparte de las no escasas semejanzas que tiene con su Pastorale. El argumento decisivo sobre la autenticidad del ars praedicandi se halla en el capítulo 684 del Terç del Crestià donde Eiximenis hace alusión expresa a un «art especial que en suma he posada en lo començament del primer libre dominical dels sermons», dirigido para que aquellos que tuviesen que predicar pudieran recordar las cosas que había que decir, finalidad expresamente señalada en una de las primeras páginas del ars praedicandi. En este sentido se pretende instruir al orador para que de un modo más eficaz pueda convencer a sus oyentes. Dicha obra responde a una línea general de manuales sobre predicación de la época. La estructura de los sermones corresponde a la división en una introducción general, introducción del tema y división de la materia. Siempre se comenzaba con un tema bíblico que propiciaba el Evangelio o la epístola del día, para seguir con una corta oración y con la introductio thematis de la explicación del sentido literal del texto y las aplicaciones morales del mismo. La tercera parte comenzaba con el enunciado de las divisiones que la integraban y el desarrollo de cada una de ellas. Los ejemplos eran muy adecuados para el auditorio y el predicador debía acompañar sus palabras con gestos que dieran mayor dramatismo a la argumentación.

Por último, indicar que el estudio de la relación de libros de la biblioteca de Francesc Eiximenis publicada hace más de treinta años es indicativo de muy poco, ya que allí se recogen una serie de libros de cuando era obispo de Elna ${ }^{14}$ y hacía bastante tiempo que había escrito la mayor parte de sus obras, al menos las más importantes de ellas. En cualquier caso, el problema de las fuentes de Eiximenis es tema complejo dentro de su obra que no ha sido resuelto ni por David J. Viera, Curt Wittlin, Jill R. Webster, Jorge E. Gracia, ni por quien escribe estas líneas en homenaje póstumo a Regina Sáinz de la Maza. El tiempo dirá y no creo que se haya dicho

\footnotetext{
${ }^{13}$ Consultar Martí de Barcelona, Fra Francesc Eiximenis, O. M (1340?-1409?), en "Estudios Franciscanos", XL (1928), pp. 477-478 y antes en la propia revista "Estudios Franciscanos", XXXVI (1925), pp. 450-452.

${ }^{14}$ Jacques Monfrin, La bibliothèque de Francesc Eiximenis (1409), en "Bibliothèque d'Humanisme et Renaissance", XXIX (1967), pp. 447-484.
} 
respecto al franciscano la última palabra de ninguna de sus obras. Nuevas técnicas de investigación en el siglo XXI permitirán descubrir muchas cosas y cuestionar en tantos puntos, no sólo los resultados alcanzados hasta ahora, sino la atribución de alguna de sus obras y la originalidad de diversos capítulos de sus escritos.

\section{RÉSUMÉ}

Étude sur les sources canoniques dans les oeuvres de Francesc Eiximenis (c. 13301409), évêque d'Elne (Lo libre de les dones, Dotzè del Crestià et Terç del Crestià). Déjà dans le Premier del Crestià il avait mentionné la papauté d'Avignon, mais sans se déclarer pour autant vraiment favorable, quoique ce sont des choses qu'il avait écrit vers les années comprises entre 1379 et 1381 . Plus tard, il eut l'occasion de changer d'idée dans De triplici statu mundi.

\section{SUMMARY}

Study of the essential juridic sources of the canonic law in Francesc Eixime is'works. The article goes over Lo libre de les dones, Dotzè del Crestià, Terç del Crestià and De triplici statu mundi. Eiximenis was a defender of the Avignon's Pontificate 